\title{
Doppler imaging of stellar surface structure
}

\section{The rapidly-rotating single K2-giant HD $31993=$ V1192 Orionis}

\author{
K. G. Strassmeier ${ }^{1, \star}$, L. Kratzwald ${ }^{2}$, and M. Weber ${ }^{1, \star}$ \\ 1 Astrophysical Institute Potsdam, An der Sternwarte 16, 14482 Potsdam, Germany \\ e-mail: mweber@aip.de \\ ${ }^{2}$ Institut für Astronomie, Universität Wien, Türkenschanzstr. 17, 1180 Wien, Austria \\ e-mail: leonidios@gmx.at
}

Received 19 January 2000 / Accepted 1 July 2003

\begin{abstract}
We present two Doppler images from two consecutive stellar rotations of the single K2-giant HD 31993. Each Doppler image is reconstructed from spectra obtained within a single stellar rotation. With its 25-day rotational period and a radius of $\approx 18$ solar radii, HD 31993 is considered a very rapidly rotating star and thus allows the application of the Dopplerimaging technique, despite the unusually long period. All maps reveal 7 isolated, predominantly low-latitude spots with a temperature difference, photosphere minus spot, of just $\approx 200 \mathrm{~K}$. No polar spot or high-latitude activity above, say, $+60^{\circ}$ is seen. A large warm feature is detected at high latitude and is believed to be real. These spots act as tracers for a cross correlation analysis and yield a clear signature of anti-solar differential surface rotation, i.e. the polar regions rotating faster than the equator, with $\alpha=0.125 \pm 0.05$ corresponding to a lap time of $\approx 200$ days. A detailed parameter study is carried out to verify the reality of the HD 31993 maps.
\end{abstract}

Key words. stars: activity - stars: imaging - stars: individual: HD31993 - stars: late-type - stars: starspots

\section{Introduction}

HD 31993 (V1192 Ori) is a bright and rapidly rotating single $\mathrm{K} 2$ giant with a rotation period of approximately 25 days. The spectral classification of K2III is originally from Bidelman \& MacConnell (1973) and has been confirmed in more detail by Fekel et al. (1986) and Strassmeier et al. (1990), who observed the star at higher spectral resolution. They also found a value for $v \sin i$ of $31 \pm 2 \mathrm{~km} \mathrm{~s}^{-1}$, which is unusually high for an evolved single K2 star. Fekel (1997) revised his earlier value to even $33.4 \pm 1 \mathrm{~km} \mathrm{~s}^{-1}$ on the basis of a more precise value for the macroturbulence, while DeMedeiros \& Mayor (1999) again found a value of $31.1 \pm 3.1 \mathrm{~km} \mathrm{~s}^{-1}$. Beavers \& Eitter (1986) determined the first radial velocities from spectra taken between 1976 and 1984 and found the star to be constant and thus suggested that HD 31993 is a single star. This was independently confirmed during the southern spectrum survey of Balona (1987) as well as by Fekel \& Balachandran (1993) and the more recent observations by DeMedeiros \& Mayor (1999).

Ultraviolet IUE data and the discovery of a strong lithium line were reported by Fekel \& Balachandran (1993), who

Send offprint requests to: K. G. Strassmeier, e-mail: kstrassmeier@aip.de

* Visiting Astronomer, National Solar Observatory, operated by the Association of Universities for Research in Astronomy, Inc. under contract with the National Science Foundation. determined a surface lithium abundance of $\log n=1.4$ (on the usual $\log n(\mathrm{H})=12$ scale) and thus drew further attention to the star. Later on, Charbonnel \& Balachandran (2000) concluded that this abundance is not abnormal for a low-mass star on the RGB and that HD 31993 appears to be still in the Li-dilution phase after the first dredge-up. They identified two distinct evolutionary episodes on the RGB in which extra mixing of Li may cause an unusual high surface abundance. Both episodes are related to the close vicinity of the bottom of the convective envelope to the hydrogen-burning shell. Rotational mixing will likely play an important role. While strong flares are also thought to be a possibility to produce surface lithium on solar-type stars (Spite et al. 1984; Ramaty et al. 2000), surface temperature inhomogeneities may be a reason for overestimating the abundances measured or may even bear the fingerprints of the dredge-up mechanism. Several authors, most recently Israelian et al. (2001), suggested that the engulfing of a Jupiter-like planet would also dredge-up lithium and angular momentum, along with other light elements like beryllium. However, the predicted Be increase for such an event is not confirmed by the observations of lithium-rich giants by Castilho et al. (1999, 2000) and also contradicts the finding of Balachandran et al. (2000) that the abundances from the resonance and excited ${ }^{7} \mathrm{Li}$ lines only agree if ${ }^{6} \mathrm{Li}$ is absent. 
However, the actual mixing process in these giants still remains unknown.

HD 31993 was observed during the Einstein extended medium-sensitivity survey and Micela et al. (1997) listed an $\mathrm{X}$-ray luminosity of $3.55 \times 10^{30} \mathrm{erg} \mathrm{s}^{-1}$, almost four orders of magnitude higher than the solar value. Earlier radio continuum data at $8.4 \mathrm{GHz}$ already suggested strong burst-like activity. Slee et al. (1987) obtained eight measurements between 198184 and found fluxes between 0 and 6 mJy. HD 31993 was also detected with IRAS (IRAS 04575+0312) in all of its four bandpasses with fluxes of $0.785 \mathrm{Jy}$ at $12 \mu \mathrm{m}, 0.18 \mathrm{Jy}$ at $25 \mu \mathrm{m}$, $<0.4 \mathrm{Jy}$ at $60 \mu \mathrm{m}$, and $<1.0 \mathrm{Jy}$ at $100 \mu \mathrm{m}$ (see Gezari et al. 1999).

The light variability was first mentioned by Lloyd-Evans $\&$ Koen (1987) from data taken in 1980. They found a period of 6.78 days with a very small amplitude of just $0.03-$ $0.05 \mathrm{mag}$ in $V$. Several seasons of photometry were analyzed by Hooten \& Hall (1990), including data from as early as 1978 , but their period analysis allowed various photometric periods between 4.8 and 29 days. Strassmeier et al. (1997a) presented APT data from three full observing seasons in 1994-96. Clear variability was only seen in two of these seasons, but only the period of 28 days was confirmed. The $v \sin i$-value together with the 28-day period interpreted as the rotation period suggested a minimum radius of $17 R_{\odot}$, consistent with the K2III classification if the inclination of the stellar rotation axis is close to $90^{\circ}$. Strassmeier et al. (1999) presented photometric data from yet another observing season (1996/97) and found an even better defined period of 26.7 days. The $V$ amplitude was still rather small, between 0.01 during December 1996 and 0.02 thereafter, and the period accordingly uncertain.

Clearly, the star is sufficiently interesting to warrant a detailed time-series analysis of its optical spectrum by means of the Doppler-imaging technique. Section 2 presents the data and describes their quality. In Sect. 3, we obtain basic astrophysical parameters for HD 31993 while Sect. 4 presents the Doppler maps from two spectral regions and for two consecutive stellar rotations and its analysis. In Sect. 5, we present a Dopplerimaging parameter study based on the input data for HD 31993. Finally in Sect. 6, we discuss and summarize our findings.

\section{Observations}

\subsection{Optical spectroscopy}

Spectroscopic observations were obtained with the McMathPierce telescope at Kitt Peak National Solar Observatory (NSO). A total of 45 spectra were taken during a 51 nights long observing run between November 3, 1996 and January 9, 1997, covering two consecutive stellar rotations. Five spectra (numbers 41 to 45 ) were not used in the present analysis because their times of observation exceeded the baseline of two stellar rotations while another four spectra (numbers 3, 10, 14 and 37) were of unacceptable quality. We used the stellar spectrograph with the $800 \times 800$ TI- 4 CCD camera at a dispersion of $0.010 \mathrm{~nm} /$ pixel and a resolving power of 38000 as judged from the width of several Th-Ar comparison-lamp lines. The observations covered only the 641-646 nm wavelength
Table 1. Observing $\log$ and radial velocities. The spectra numbers 1-19 are used for the first Doppler image, the spectra No. 20-40 for the second Doppler image.

\begin{tabular}{rllll}
\hline \hline No. & $\begin{array}{l}\text { HJD } \\
(2450000+)\end{array}$ & $\begin{array}{l}\text { phase } \\
\left({ }^{\circ}\right)\end{array}$ & $\begin{array}{l}v_{\mathrm{r}} \\
\left(\mathrm{km} \mathrm{s}^{-1}\right)\end{array}$ & $\begin{array}{l}\sigma_{\mathrm{vr}} \\
\left(\mathrm{km} \mathrm{s}^{-1}\right)\end{array}$ \\
\hline 1 & 390.955 & 309.5 & 8.8 & 2.4 \\
2 & 391.896 & 322.9 & 12.0 & 2.4 \\
3 & 392.864 & 336.7 & $5.1:$ & 3.2 \\
4 & 393.861 & 350.9 & 16.2 & 2.5 \\
5 & 394.827 & 4.6 & 11.5 & 2.6 \\
6 & 395.888 & 19.7 & 12.4 & 2.2 \\
7 & 396.877 & 33.8 & 10.8 & 2.4 \\
8 & 399.991 & 78.1 & 12.8 & 2.4 \\
9 & 400.922 & 91.3 & 12.5 & 2.1 \\
10 & 401.927 & 105.6 & 14.1 & 2.6 \\
11 & 404.927 & 148.3 & 10.9 & 2.6 \\
12 & 405.852 & 161.5 & 10.4 & 3.7 \\
13 & 406.862 & 175.9 & 10.8 & 2.7 \\
14 & 408.877 & 204.5 & 16.3 & 2.9 \\
15 & 411.876 & 247.2 & 12.8 & 2.7 \\
16 & 412.817 & 260.6 & 11.7 & 2.7 \\
17 & 413.779 & 274.3 & 12.7 & 3.3 \\
18 & 415.877 & 304.1 & 13.1 & 2.4 \\
19 & 416.882 & 318.4 & 12.3 & 2.4 \\
\hline 20 & 418.936 & 347.7 & 12.1 & 2.7 \\
21 & 419.879 & 1.1 & 12.8 & 3.0 \\
22 & 420.919 & 15.9 & 12.4 & 2.7 \\
23 & 421.915 & 30.0 & 12.6 & 3.1 \\
24 & 422.902 & 44.1 & 12.7 & 2.2 \\
25 & 424.987 & 73.8 & 11.1 & 2.7 \\
26 & 425.908 & 86.9 & 12.4 & 2.7 \\
27 & 426.914 & 101.2 & 12.4 & 2.8 \\
28 & 428.940 & 130.0 & 12.5 & 1.7 \\
29 & 429.900 & 143.7 & 11.7 & 2.8 \\
30 & 430.912 & 158.1 & 13.2 & 2.3 \\
31 & 431.779 & 170.4 & 11.0 & 2.6 \\
32 & 432.866 & 185.9 & 11.5 & 2.8 \\
33 & 433.800 & 199.2 & 12.3 & 3.1 \\
34 & 434.882 & 214.6 & 12.2 & 3.1 \\
35 & 436.848 & 242.5 & 8.5 & 3.0 \\
36 & 437.854 & 256.9 & 12.6 & 2.5 \\
37 & 438.858 & 271.2 & 14.3 & 2.8 \\
38 & 439.906 & 286.1 & 9.7 & 2.6 \\
39 & 440.848 & 299.5 & 11.2 & 3.0 \\
40 & 441.858 & 313.9 & 12.1 & 2.5 \\
\hline & & & & \\
\hline
\end{tabular}

range due to a cosmetic defect on the detector. It thus included only two major spectral lines suitable for Doppler imaging (Ca I 643.9 and Fe I 643.0). The average signal-to-noise (S/N) ratio of our spectra is $\approx 200: 1$.

Data reductions were performed with the NOAO/IRAF software package and followed our standard procedure for coudé spectra (see, e.g., Weber \& Strassmeier 1998). Nightly observations of the radial-velocity standard $\alpha$ Ari were used to obtain the radial velocities for HD 31993, except for one night where $\beta$ Gem was used instead. We adopted $v_{\alpha \text { Ari }}=$ $-14.51 \mathrm{~km} \mathrm{~s}^{-1}$ and $v_{\beta \mathrm{Gem}}=+3.23 \mathrm{~km} \mathrm{~s}^{-1}$ (Scarfe et al. 1990). Table 1 lists the spectra and its radial velocities. 


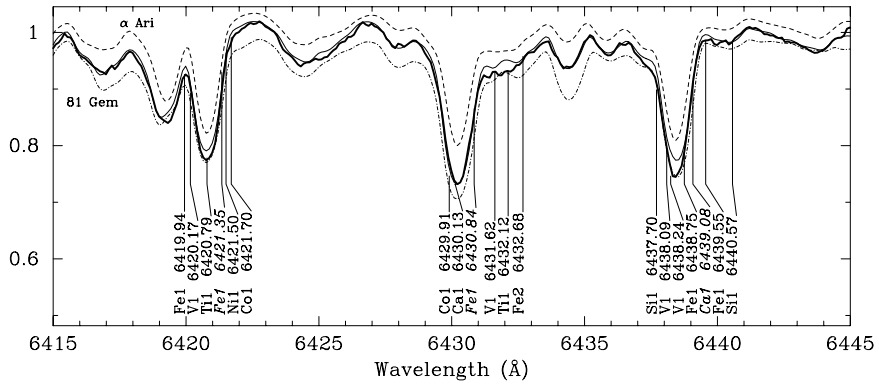

Fig. 1. An example of a spectrum used for the Doppler-imaging analysis in this paper (thick line). The spectral lines of interest are marked. Also shown is a comparison with spectra of the KIII M-K standard stars $\iota$ Hya (K2.5III - thin full line), $\alpha$ Ari (K2III - dashed line), and $81 \mathrm{Gem}$ (K4III - dash-dotted line). The best match is found with $\iota$ Hya.

\subsection{Photometry}

Photometric data were collected between 1993-1998 with the Amadeus 0.75-m automatic photoelectric telescope (APT) of the University of Vienna located at and operated by Fairborn Observatory in Arizona (Strassmeier et al. 1997b). The telescope was equipped with Johnson-Cousins $V, R$ and $I$ filters. Altogether, $60 V(R I)_{C}$ measurements were obtained during the spectroscopic campaign, each of them being the mean of three readings of the variable-minus-comparison brightness. HD 32191 was used as the comparison star $(V=8.53)$ and HD 32073 as the check star.

All photometric and spectroscopic data are phased with the photometric period determined in Sect. 3 and from a time of light-curve maximum,

$\mathrm{HJD}=2450369.2+25.3 \times E$.

\section{Stellar parameters of HD 31993}

We first verify the star's spectral classification by a detailed comparison of its optical spectrum in the wavelength range 641-646 nm with spectra of M-K standard stars. In this procedure, we synthesize a reference spectrum from a standardstar spectrum by shifting it in radial velocity and rotationally broaden it to match the spectrum of HD 31993. This spectrum is then subtracted from the observed one and its difference minimized by means of a least-squares algorithm using a modified program by Huenemoerder \& Ramsey (1987). Figure 1 shows the results. The best fit to the spectrum was achieved with $\iota$ Hya (K2.5III, $B-V=1$ m 34 ). Because the dereddened $B-V$ index of HD 31993 is bluer by 0 . 15 than that of $\iota$ Hya but redder by 0.05 than for $\alpha$ Ari $(B-V=1 \mathrm{~m}$. 15$)$, we believe that HD 31993 is most likely slightly warmer than K2.5, and we conclude that it is best classified as a K2III star.

The total of 40 high-resolution spectra were also used to determine radial velocities (listed in Table 1) by means of cross correlations with the radial-velocity standard $\alpha$ Ari (K2III). Its mean value is $12.2 \pm 1.0(\mathrm{rms}) \mathrm{km} \mathrm{s}^{-1}$, in good agreement with previously published values. A periodogram analysis did not result in any significant periodicity. We conclude that HD 31993 is indeed a single star.

Another important astrophysical parameter is the stellar rotational period. Because HD 31993 is a single star, we must a. $1996 / 97$ V-light curve

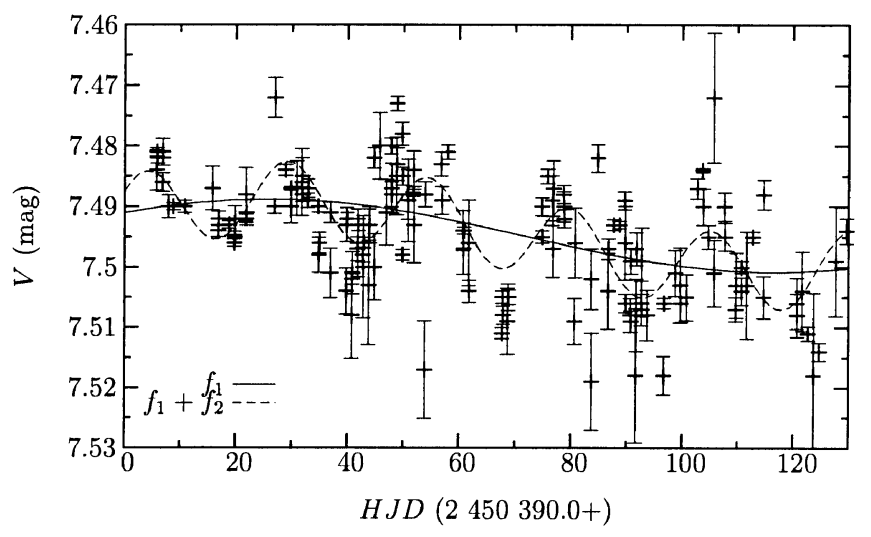

\section{b. $1996 / 97$ periodogram}

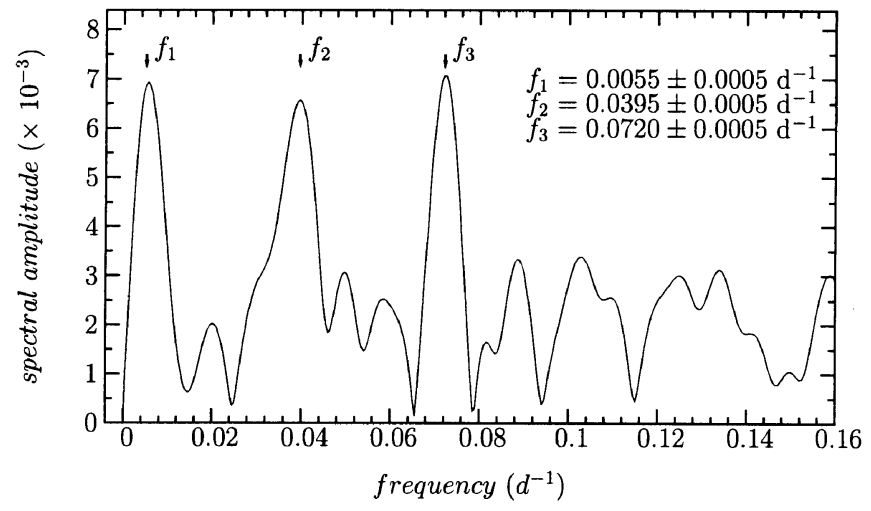

Fig. 2. a) APT $V$-light curve for the observing season 1996/1997 and b) its periodogram. The lines are the results from combined sine-curve fits with $f_{1}$ and $f_{2}$. The errors quoted are internal errors. The spectroscopic observations in this paper were obtained within the first two photometric cycles between $2450390-441$.

solely rely on the period from the time-series analysis of its photometry. Previously claimed periods from broad-band photometry range from 4.8 to 29 days (see Sect. 1). Figure 2 presents our $V$-band data for the 1996/97 observing season and the results from a Fourier analysis with optimized $\chi^{2}$ fitting (Sperl 1998). Three frequencies appeared with comparable O-C's corresponding to the following periods: $182 \pm$ 18 days $\left(f_{1}\right), 25.3 \pm 0.3$ days $\left(f_{2}\right)$, and $13.9 \pm 0.1$ days $\left(f_{3}\right)$. Notice that the peak-to-peak amplitude was just 0.012 in $V$ and that the overall system brightness was fading within the season by 0.01 . This fading resulted in the pseudo-periodic 182-day sine curve shown in Fig. 2a and confirms the long-term trend of the average brightness already indicated in Fig. 9 in Strassmeier et al. (1997a). The 13.9-day period seems to be the $2 \mathrm{f}$ harmonic of the true $\approx 26$-day period found in earlier studies and would result in a minimum radius of $9 R_{\odot}$, in contradiction to the radius from the Hipparcos distance. Thus, the 25.3-day period seems to fit the overall astrophysical parameters much better, and is in agreement with previous period determinations. We adopt it to be the stellar rotation period.

A minimum radius $R \sin i$ of $16.6 \pm 0.3 R_{\odot}$ is computed from the rotational period of 25.3 days and the projected rotational velocity of $33.2 \pm 0.5 \mathrm{~km} \mathrm{~s}^{-1}$. With $i=65^{\circ} \pm 10^{\circ}$ from 


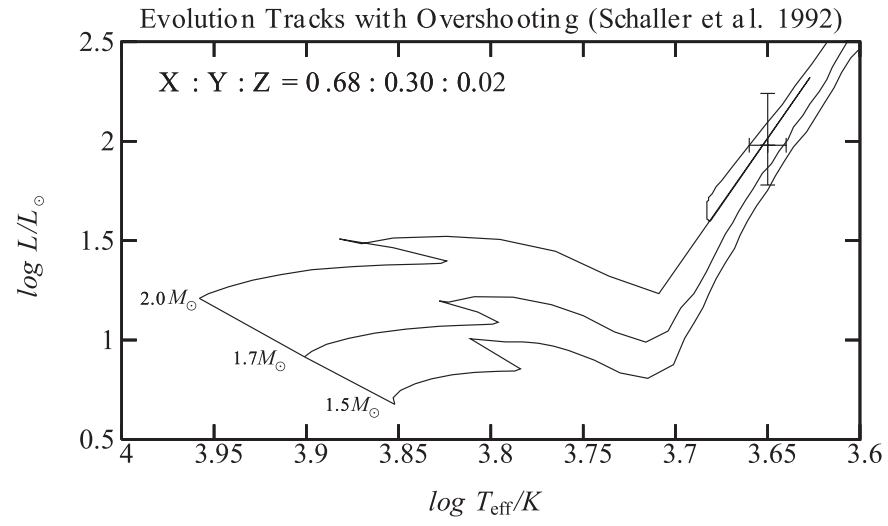

Fig. 3. The position of HD 31993 in the H-R diagram (cross). The lines are the "overshooting tracks" for masses of 1.5, 1.7, and $2.0 M_{\odot}$ from Schaller et al. (1992).

the tomographic parameter study in the next section, $R$ is then $18.3_{-1.1}^{+2.0} R_{\odot}$. This value is in agreement with the expected radius for a normal K2 giant (e.g. Gray 1992) as well as with the $16_{-4}^{+6} R_{\odot}$ computed from the distance and the effective temperature. The Hipparcos parallax of $4.20 \pm 1.09$ milli-" (ESA 1997) combined with the brightest $V$ magnitude observed so far, $V=7 \mathrm{~m}$. 48 (this paper, dereddened value: $V_{0}=7 \mathrm{~m} .28$ ), result in an absolute visual brightness of $+0.40_{-0.65}^{+0.50}$.

With an assumed reddening of 0.2 , the Tycho $B-V$ of 1 . 254 indicates an effective temperature of $4500 \mathrm{~K}$ (according to the calibration of Flower 1996). The bolometric magnitude of -0 . 20 converts to a luminosity of $93_{-34}^{+76} L_{\odot}$ (with $M_{\text {bolø }}=+4.72$ ). The position in the theoretical H-R diagram (Fig. 3) then shows that HD 31993 is beyond the base of the giant branch and could even be in or at least very near the helium-core burning phase at the ascend to the asymptotic giant branch. A comparison with the evolutionary tracks from Schaller et al. (1992) for solar metallicity $(Z=0.02)$ implies a mass of $1.9 \pm 0.3 M_{\odot}$ and an age of $\approx 1-2 \mathrm{Gyr}$.

Table 2 summarizes the astrophysical parameters for HD 31993.

\section{Doppler imaging analysis}

\subsection{The line profile inversion code TempMap}

TempMap performs an LTE spectrum synthesis by solving the equation of transfer through a set of ten Kurucz (1993) model atmospheres, at all aspect angles, and for a given set of chemical abundances. Simultaneous inversions of up to seven spectral lines, as well as two photometric bandpasses, were carried out with a maximum-entropy regularization. The number of iterations was set to 15 , which proved to be sufficient for a good convergence and to reproduce the $\mathrm{S} / \mathrm{N}$ of the data (for a recent example see Strassmeier \& Rice 2003). The computations were performed on a Celeron-driven Linux PC and required $30 \mathrm{~min}$ of CPU time for each Doppler map. A more detailed description of the TempMap code (Rice et al. 1989) and additional references regarding our line-profile inversion technique can be found in Rice et al. (2002) and Rice \& Strassmeier (2000) and previous papers of this series.
Table 2. Astrophysical data for HD 31993.

\begin{tabular}{ll}
\hline \hline Parameter & Value \\
\hline Classification & K2III, single star \\
Distance (Hipparcos) & $238_{-49}^{+83} \mathrm{pc}$ \\
Luminosity, $L$ & $93_{-34}^{+76} L_{\odot}$ \\
$\log g$ & $2.5_{-0.4}^{+0.2}$ \\
Mass & $1.9 \pm 0.3 M_{\odot}$ \\
$T_{\text {eff }}$ & $4500 \pm 50 \mathrm{~K}$ \\
$(B-V)_{\text {Hipparcos }}$ & $1.254 \pm 0.015 \mathrm{mag}$ \\
$(V-I)_{\text {Hipparcos }}$ & $1.21 \pm 0.02 \mathrm{mag}$ \\
$v \sin i$ & $33.2 \pm 0.5 \mathrm{~km} \mathrm{~s}^{-1}$ \\
Inclination, $i$ & $65 \pm 10^{\circ}$ \\
Period, $P_{\text {rot }}$ & $25.3 \pm 0.3$ days \\
Radius, $R$ & $18.3_{-1.1}^{+2.0} R_{\odot}$ \\
Microturbulence, $\xi$ & $2.0 \mathrm{~km} \mathrm{~s}^{-1}$ \\
Macroturbulence, $\zeta_{\mathrm{R}}=\zeta_{\mathrm{T}}$ & $3.0 \mathrm{~km} \mathrm{~s}^{-1}$ \\
Abundance, Fe & $\approx 7.7$ \\
Abundance, Ca & $\approx 6.5$ \\
\hline
\end{tabular}

\subsection{Atomic line-data input}

We adopted the atomic parameters that we already used in previous studies of K giants (e.g. Strassmeier 1999). By default, the Castilho et al. (2000) photospheric abundances were adopted unless otherwise noted. HD 31993 appears to be significantly overabundant in metals, e.g. by 0.12 dex for $\mathrm{Fe}$ or 0.2 dex for Ca and even 0.9 dex for Co (always with respect to the solar abundances given by Grevesse \& Sauval 1998, 1999).

\subsection{Two consecutive Doppler images for late 1996}

We employ the CaI line at $643.9 \mathrm{~nm}$ and the Fe I line at $643.0 \mathrm{~nm}$ as the main mapping lines. As mentioned in previous papers in this series (e.g. Kővári et al. 2001), the Ca I643.9 line is quite sensitive to temperature gradients at the photospheric temperatures of early $\mathrm{K}$ giants. Cooler regions on the stellar surface will appear less pronounced in Ca-based images than in Fe-images because inside a spot, i.e. in the regions with less continuum contribution, the $\mathrm{Ca}$ line has a relatively larger equivalent width and hence the bump produced by the spot in the Ca profiles appears less significant.

Figures 4 and 5 show the results for the two consecutive stellar rotations for the Ca I-643.9 and the Fe I-643.0 line region, respectively. The identifications in these figures allow a more convenient comparison of individual features from one rotation to the next and for both lines separately. However, their assignment is premature.

\subsubsection{Rotation \#1}

The main feature of the maps from both spectral lines is the large number of cool, low-latitude $\left(0-40^{\circ}\right)$ spots or spot groups that seem to be distributed at nearly equidistant separations around the star. No polar spot is obvious. We identify up to 7 individual spots from both the $\mathrm{Ca}$ and the Fe map (dubbed $\mathrm{A}-\mathrm{G}$ in Fig. 4). Both chemical species also agree in their small temperature contrast with respect to the photospheric temperature 


\section{a. Ca-map rotation \#1}
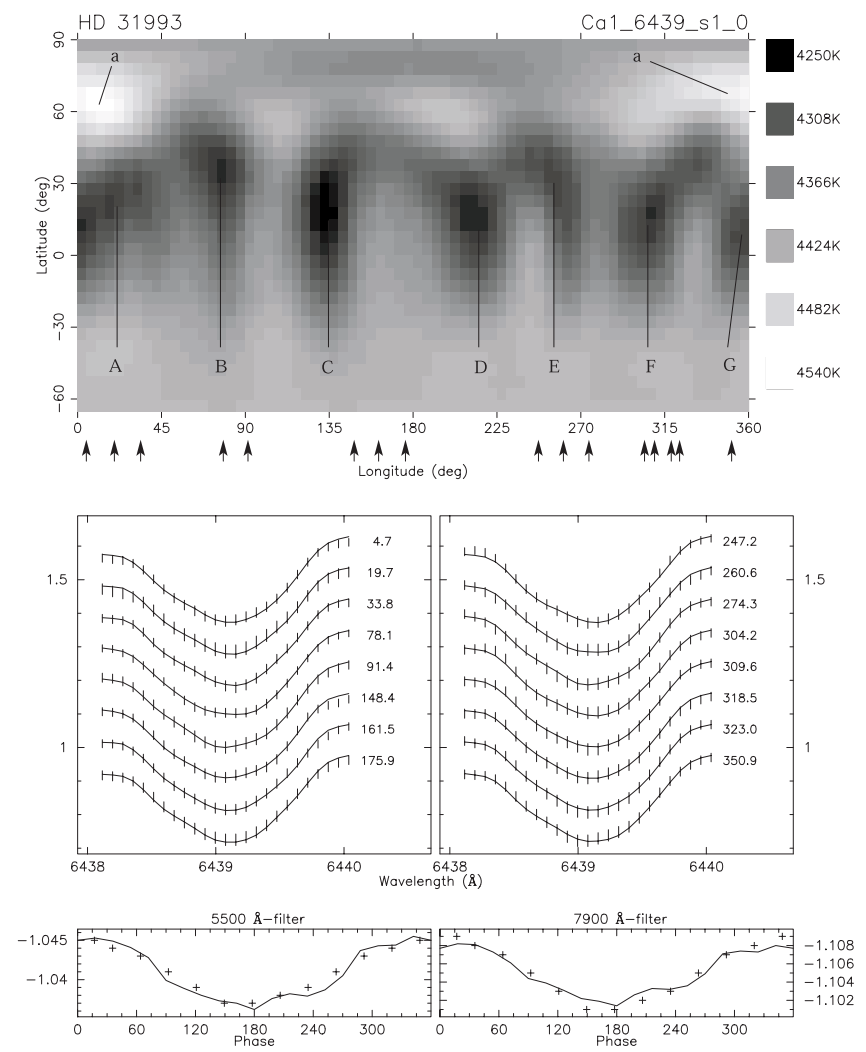

b. Ca-map rotation \#2
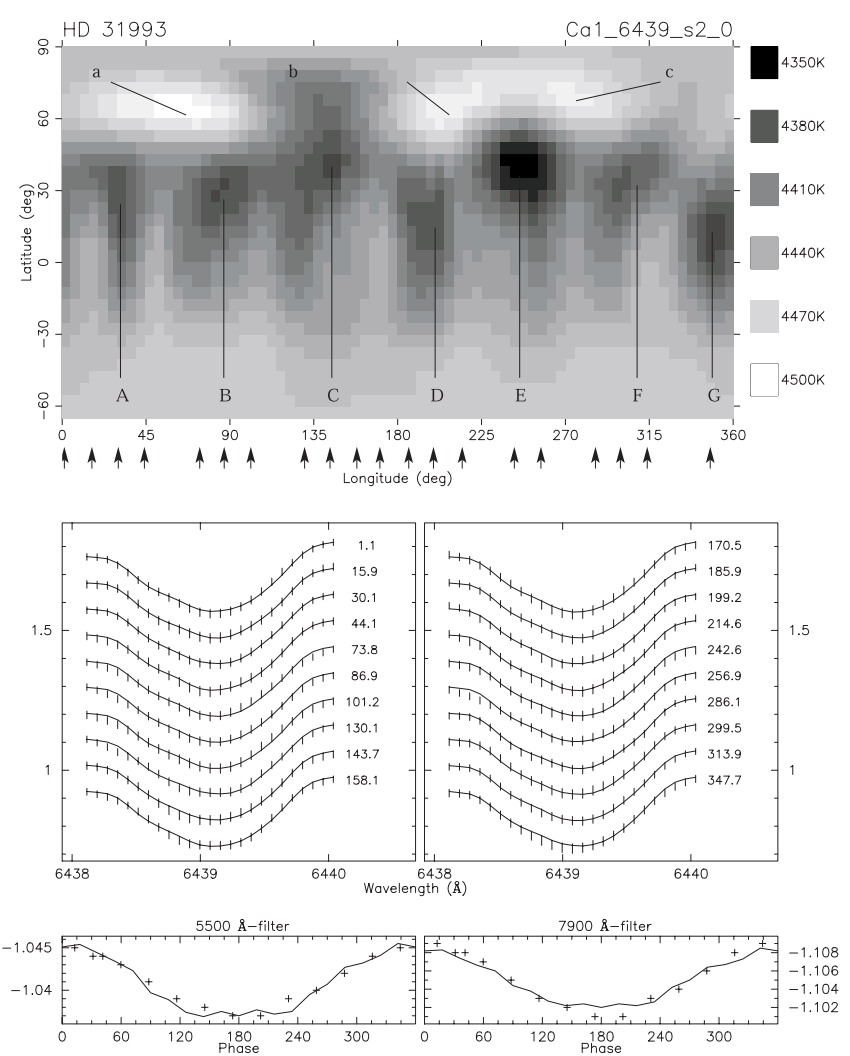

Fig. 4. Ca I-643.9 results for a) rotation \#1 and b) rotation \#2. Top panels: pseudo Mercator projection with identification of individual features. Bottom panels: line profile and photometric data and the respective fits. The arrows below the top panels indicate the phases of the spectroscopic data while the representation of the line-profile point as bars indicate its $\pm 1 \sigma$ deviation. Notice that the light-curve points in the lower panels are equidistant points from the Fourier fit to the actual data as shown in Fig. 2a.

of $4500 \mathrm{~K}$. The Ca map spans the range from 4250 to $4540 \mathrm{~K}$, i.e. $\Delta T=290 \mathrm{~K}$, while the $\mathrm{Fe}$ map spans the range 4340 to $4520 \mathrm{~K}$, i.e. just $\Delta T=180 \mathrm{~K}$. Compared to previous maps of active giants, this is the lowest contrast ever detected (but see Sect. 6). The parameter study in Sect. 5 is an attempt to verify or falsify the reality of this result.

Another feature that appears in the maps from both spectral lines is an elongated warm region at high $\left(60-70^{\circ}\right)$ latitude around zero longitude. This feature is called $a$ in Table 3 and marked in the Ca map \#1 in Fig. 4a and in the Fe map \#1 in Fig. 5a. It is of rather weak contrast, $\approx 40 \mathrm{~K}$ above the effective temperature, but appears well constrained from the data coverage and we consider it real (see also the reconstruction tests with hot spots in Rice \& Strassmeier 2000).

\subsubsection{Rotation \#2}

The maps from the second data set basically verify the surface morphology from the previous rotation. The $\mathrm{Ca}$ map is reconstructed with 7 low-latitude spots and up to 3 warm regions at high latitude, while the Fe map has also 7 low-latitude cool spots but only one warm region. The respective latitudes and longitudes of these features are summarized in Table 3. The overall temperature ranges are also comparable to the first rotation; 4350-4500 $(\Delta T=150 \mathrm{~K})$ in the Ca map, and 4320$4500(\Delta T=180 \mathrm{~K})$ in the Fe map. Again, the cool regions are on average only $150-180 \mathrm{~K}$ cooler than the effective temperature and the warm region(s) only about $30 \mathrm{~K}$ warmer than their averaged surrounding. Notice that the warm feature, $a$, at $\ell \approx 0^{\circ}$ from the first rotation also appears in the second rotation but shifted in longitude by $\Delta \ell \approx 50^{\circ}$. It appears in the reconstruction from both spectral lines, while features $b$ and $c$ from the Ca profiles do not repeat from the Fe profiles. We thus consider $a$ real but $b$ and $c$ spurious.

\subsection{Differential surface rotation and spot evolution}

We cross correlate the maps from the two consecutive rotations and interpret the residual, latitude-dependent phase shifts as differential surface rotation (see Collier Cameron 2002; Weber \& Strassmeier 2001), and/or as short-term spot evolution in case the residual pattern is non-systematic (e.g. as on $\sigma$ Gem; Kôvári et al. 2001). The final cross-correlation function maps (ccf-maps) for the two rotations are displayed in Fig. 6, along with our derived differential-rotation law.

In this paper, we carry out a more elaborate statistical approach to determine differential surface rotation. The aim is to obtain a more quantitative measure of the uncertainties of the 


\section{a. Fe-map rotation \#1}
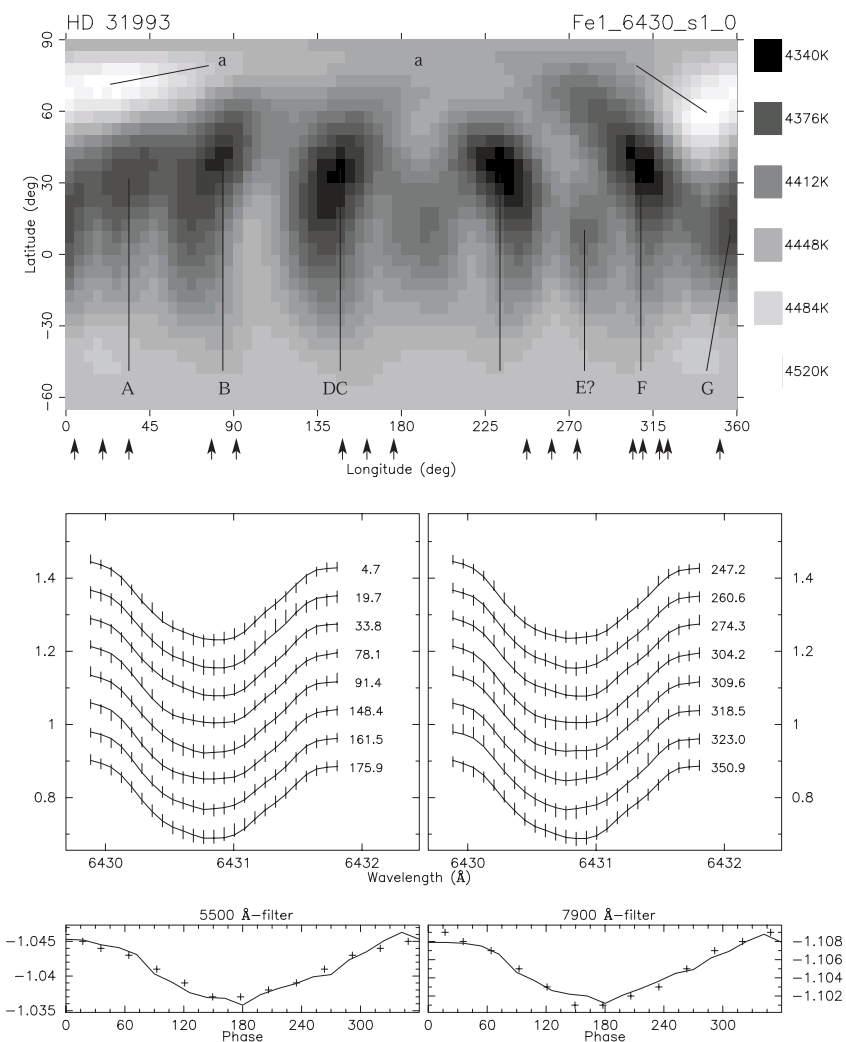

\section{b. Fe-map rotation \#2}
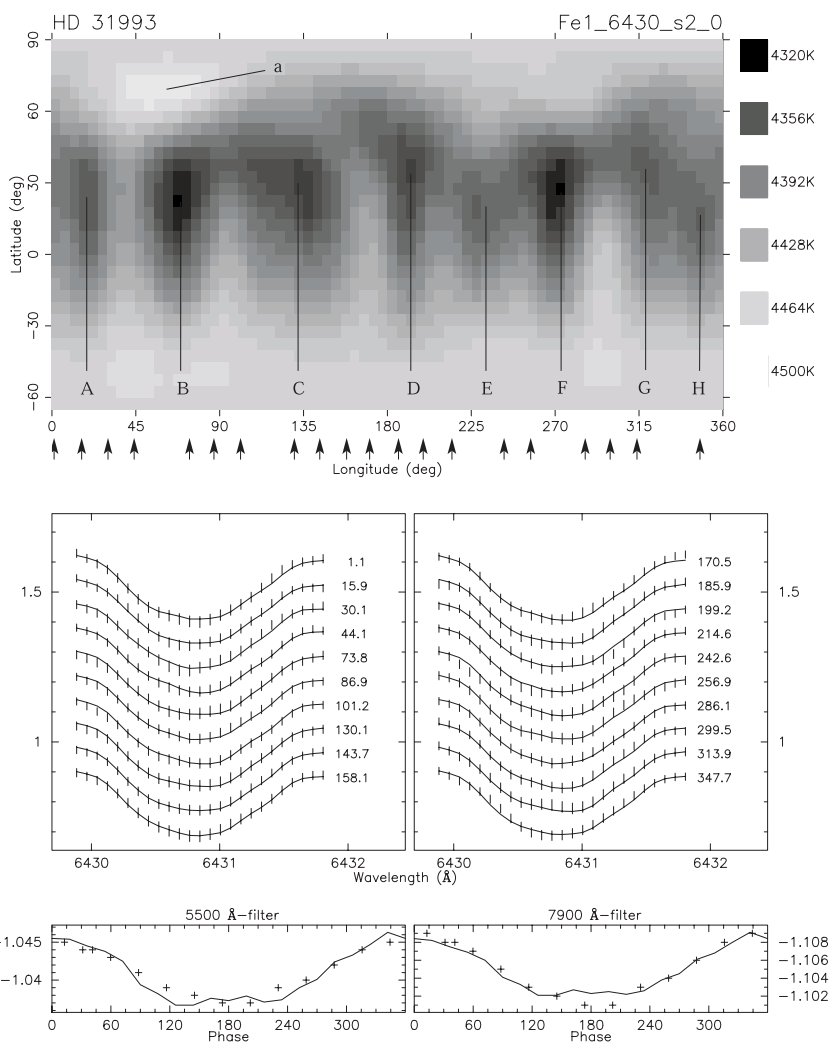

Fig. 5. Fe I-643.0 results for a) rotation \#1 and b) rotation \#2. Otherwise as in Fig. 4.

Table 3. Longitudes and latitudes of surface features in degrees. The identifications (ID) follow Figs. 4 and 5. Capital letters refer to cool spots, lower-case letters to warm spots. Features $b$ and $c$ are not considered real (see text).

\begin{tabular}{lllll}
\hline \hline \multirow{2}{*}{ ID } & \multicolumn{2}{c}{ Rotation \#1 } & \multicolumn{2}{c}{ Rotation \#2 } \\
& $\mathrm{Ca}$ & $\mathrm{Fe}$ & $\mathrm{Ca}$ & $\mathrm{Fe}$ \\
\hline $\mathrm{A}$ & $12 / 20$ & $33 / 30$ & $30 / 25$ & $20 / 25$ \\
$\mathrm{~B}$ & $75 / 35$ & $80 / 40$ & $83 / 30$ & $60 / 28$ \\
$\mathrm{C}$ & $133 / 20$ & $143 / 33$ & $143 / 40$ & $133 / 32$ \\
$\mathrm{D}$ & $213 / 16$ & $233 / 35$ & $197 / 20$ & $195 / 38$ \\
$\mathrm{E}$ & $253 / 36$ & $280 / 10$ & $245 / 40$ & $230 / 22$ \\
$\mathrm{~F}$ & $308 / 16$ & $310 / 35$ & $308 / 30$ & $270 / 30$ \\
$\mathrm{G}$ & $353 / 10$ & $353 / 15$ & $350 / 15$ & $330 / 25$ \\
$\mathrm{H}$ & & & $\ldots$ & $348 / 14$ \\
$\mathrm{a}$ & $12 / 66$ & $0 / 70$ & $58 / 65$ & $55 / 70$ \\
(b) & $\ldots$ & $\ldots$ & $205 / 60$ & $\ldots$ \\
(c) & $\ldots$ & $\ldots$ & $278 / 70$ & $\ldots$ \\
\hline
\end{tabular}

longitudinal shifts (other than by analyzing the FWHM of the cross-correlation peaks) and get a better handle on the internal error of the differential rotation parameter. The approach is based on a bootstrap Monte-Carlo procedure. For each of the four individual data sets - $\mathrm{Ca}$-line and $\mathrm{Fe}$-line region, and for the two rotations - we computed 50 maps from 50 randomly chosen (different) combinations of observational phases. For rotation \#1 a minimum of ten phases and for rotation \#2

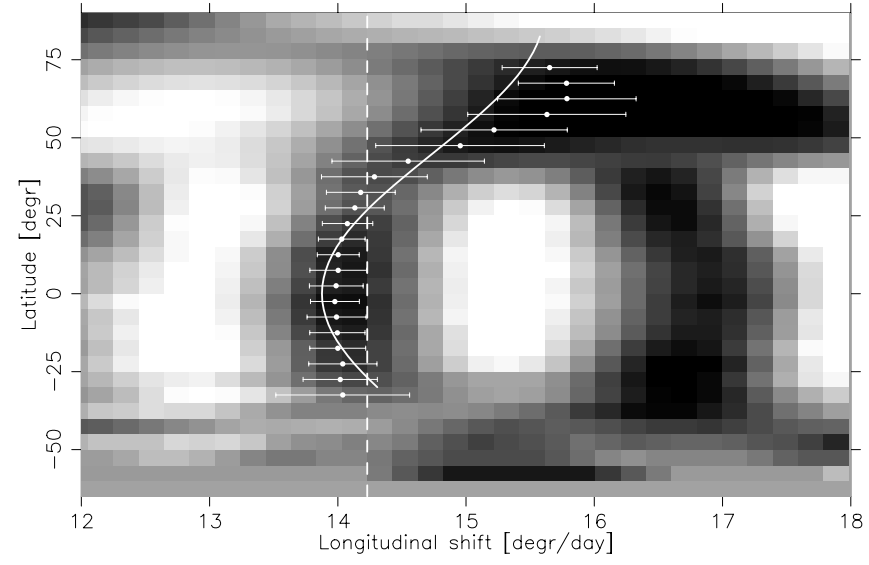

Fig. 6. The cross-correlation function (ccf) from the two independent consecutive Doppler images. The $x$-axis plots the longitudinal shifts in degrees per day, the $y$-axis shows latitude. The grey scale represents the correlation coefficient in the sense that black is perfect correlation $(=1)$ and white no correlation $(=0)$. The dots with error bars are the fits to the peaks of the ccfs per latitude bin. The vertical line is the rotational period of 25.3 days and the curve is the differential-rotation fit weighted by the FWHM of the ccf peak. An anti-solar differential rotation seems evident.

a minimum of 15 phases were adopted. Then we averaged the $\mathrm{Ca}$ - and Fe-line maps (with equal weight) and obtained 50 average surface maps for each rotation. With these average maps 50 cross-correlation functions were computed using IRAF's fxcor subroutine. We then searched for a correlation 
peak in each latitude strip (for a description of that procedure see Paper V by Weber \& Strassmeier 1998). These peaks were then fitted with a quadratic but anti-solar differential-rotation law of the form

$\Omega(b)=\Omega_{0}+\Omega_{1} \sin ^{2} b$

for each of the 50 combinations weighted with the FWHM of the corresponding autocorrelation-ccf peak, resulting in 50 values for $\Omega_{0}$ and $\Omega_{1}$ ( $b$ is the surface latitude, $\Omega_{0}$ the angular velocity of the stellar equator). The averaged values from these fits are

$\Omega_{0}=13.87 \pm 0.22 ; \quad \Omega_{1}=1.73 \pm 0.67$,

based on the latitude range $-30^{\circ}$ to $+75^{\circ}$, in steps of $5^{\circ}$ (the "error" bars are standard deviations). Figure 6 shows the fit as a curved line. HD 31993 exhibits strong anti-solar differential rotation with the polar regions rotating faster than the equatorial region. The differential-rotation coefficient $\alpha=\left(\Omega_{\text {equ. }}-\right.$ $\left.\Omega_{\text {pole }}\right) / \Omega_{\text {equ. }}=\Omega_{1} / \Omega_{0}$ is $0.125 \pm 0.050$ and the time the equatorial regions lap the polar regions is $\approx 200$ days. This is stronger differential rotation than on the similar K0-giant KU Pegasii (Weber \& Strassmeier 2001) but still significantly weaker than for the Sun (the corresponding solar value is $\approx 130$ days) or for the ZAMS star AB Dor (Collier Cameron \& Donati 2002; $\approx 110$ days).

\section{A tomographic parameter study for HD 31993}

One of the results of the TEMPMAP test study by Rice \& Strassmeier (2000) was that the "quality" or "reliability" of a Doppler image depends mostly on the external error budget of the data once an $\mathrm{S} / \mathrm{N}$ of $\approx 300: 1$ has been reached. Additionally, the LTE treatment of plane-parallel non-dynamic model atmospheres in the radiative-transfer solution in TEMPMAP together with an unrealistic description of turbulent convection (and many other approximations) start to play an important role in the local line-profile fitting once $\mathrm{S} / \mathrm{N}$ of $1000: 1$ is encountered. Although Rice \& Strassmeier (2000) generated artificial data with various levels of external errors such as continuumslope changes or various levels of spectrograph stray light, their image recoveries were always based on "ideal data" through forward computations from plane-parallel ATLAS-9 models in LTE and with MLT-type convection. In the following tests, we will use the real data of HD 31993 with all their external unknowns and uncertainties but under the assumption that the final reconstructions in Sect. 4.3 indeed represent the true surface image. This allows us to confirm the overall quality, and thus reliability, of our results in the previous Sect. 4.

"Quality" criteria in these tests are the overall $\chi^{2}$ of the fits to the data, the existence of systematic surface artifacts such as bright and dark bands or very steep temperature gradients, the minimum and maximum temperatures recovered and their difference $\Delta T=T_{\max }-T_{\min }$. Due to the large number of tests, we present here only a few relevant examples and refer the reader to the thesis of Kratzwald (2003) for further details. Figure 7 is an example of the recovery from the Fe I 643.0-nm line (first stellar rotation) for three cases with decreasing phase coverage.

\subsection{Rotational period and phase coverage}

Test inversions of the data when phased with periods between 25.0 to 27.0 days (the period range most likely from the Fourier analysis of the photometry), showed practically no differences of its surface structure and we conclude that even an uncertainty of \pm 1 day will not affect our images of HD 31993 .

The phase coverage for both consecutive rotations is very good - 16 and 20 spectra, respectively - but still not fully equidistant. To estimate the impact of imperfect phase coverage (for the first rotation only), we removed every second spectrum and reran the inversions until only three profiles were left (Fig. 7). While the case with 9 profiles showed only morphological changes below $\pm 20 \mathrm{~K}$, that would not be interpreted significant anyway, the case with 5 profiles showed already a severe artefact of a bright latitudinal band near $180^{\circ}$. The case with 3 profiles could not fit the photometry anymore (left at same weight). It also missed the spot near a longitude of $45^{\circ}$ and produced two bright longitudinal bands at $180^{\circ}$ and $270^{\circ}$. Nevertheless, it basically still recovered the large spots. This makes us confident that the original data of HD 31993 with 16 and 20 observations, respectively, were sufficient for an excellent reconstruction within the capabilities of the technique.

\subsection{Regularizing parameters}

TEMPMAP contains a number of switches that are usually kept at empirically determined values. Kratzwald (2003) identified the most important ones to be the number of iterations in the inversion loop, the "smoothing" value $\alpha$ of the regularizing functional $^{1}$ (see, e.g. Piskunov \& Rice 1993; Rice 2002), and the relative weight of the photometric data with respect to the line profiles. While the reconstructions of HD 31993 with 5, 10, 20 , and 30 iterations revealed practically no noticeable differences for a low to medium strong regularizing value, there are converging problems for high regularizing values $(\alpha \geq 10)$. This is not surprising because very strong regularization tends to underepresent the data. However, a series of simulations with varying $\alpha$ showed that the recovered surface morphology remains practically identical but that the overall temperature levels are altered. Going from $\alpha=1$ to $\alpha=12$, the minimum temperature increases from $4270 \mathrm{~K}$ to $4350 \mathrm{~K}$ while the maximum temperature decreases from $4670 \mathrm{~K}$ to $4500 \mathrm{~K}$. Given the moderate $\mathrm{S} / \mathrm{N}$ and resolution of the present NSO data of HD 31993 , the simulations showed the most consistent recovery at a fairly high value of the regularizing functional of $\alpha=9$, which was used for the final maps in Figs. 4 and 5.

It is also interesting to note that for high $\alpha$ the Tikhonov regularization resulted in a $15 \%$ better $\chi^{2}$ statistics $^{2}$ than the maximum-entropy (ME) functional, while for low $\alpha$ the results are practically identical. Similar was already mentioned by Piskunov et al. (1990). However, the better- $\chi^{2}$ Tikhonov map resulted both in a higher maximum and in a lower minimum temperature, with spots generally showing a steeper

\footnotetext{
1 Not to be confused with the differential-rotation parameter $\alpha$ in Sect. 4.4 .

2 The misfit between the observations and the model as defined in Rice \& Strassmeier (2000).
} 


\section{Test inversions with limited number of line profiles}
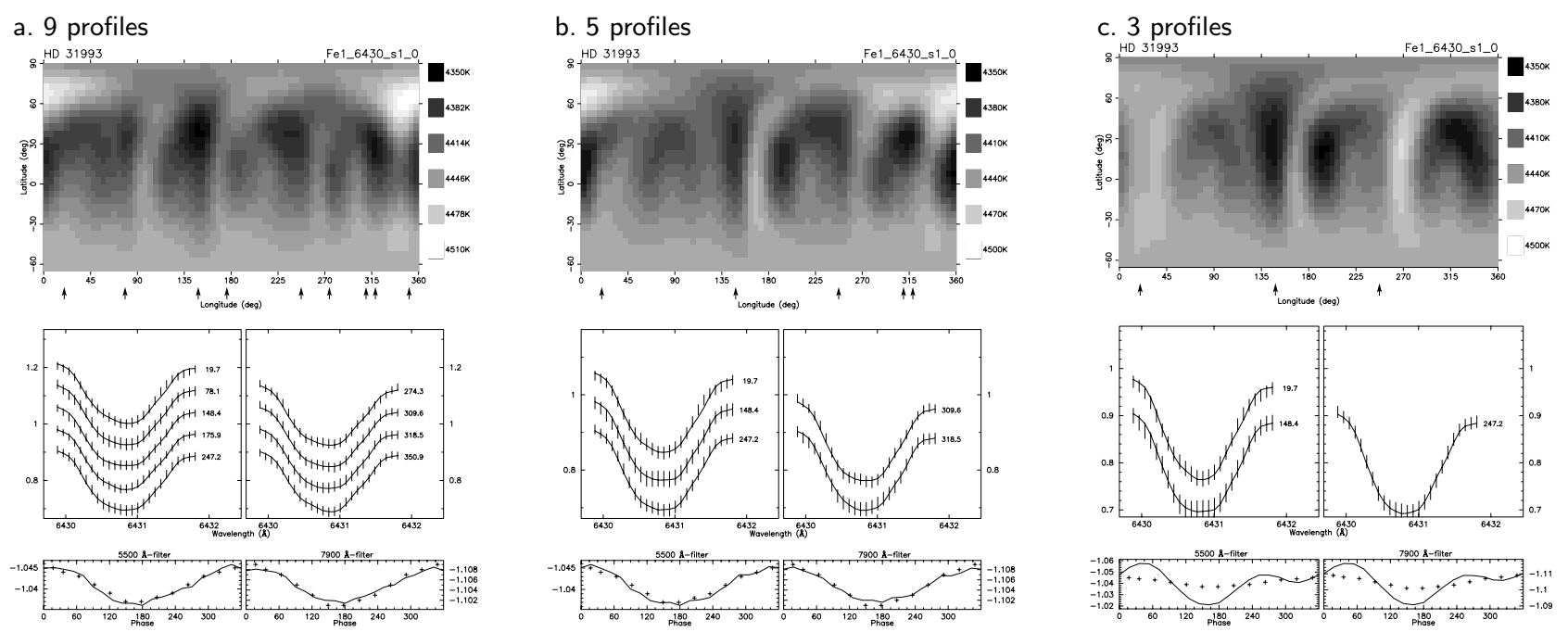

Fig. 7. Example reconstructions from the parameter study. The images show the results from three trial inversions of the Fe I 643.0-nm line from rotation \#1 but with a) 9, b) 5, and c) 3 line profiles (the nominal number of profiles was 16, see Fig. 5a). The case with 5 profiles still recovers the spots correctly, while the inversion with 3 profiles got stuck in one of the many local $\chi^{2}$ minima and also failed to reproduce the photometry.

temperature gradient than in the respective ME map with lower $\chi^{2}$. At this stage, simultaneous multi-color photometry helps to constrain the temperature range. To test its impact, we changed the relative weight of the photometry from 0 (no weight) to $0.3,0.5$, and 0.8 (for various regularizing values, respectively). The test images showed indeed a smaller temperature range in case photometry is included, but - especially for HD 31993 - had practically no impact on the map itself because the observed photometric amplitude was rather tiny (full $V$ amplitude of just 0.008 in early December 1996). Not surprisingly though, the smaller the photometric weight the better the overall $\chi^{2}$ became.

\subsection{Projected rotational velocity}

Our best value for the projected rotational velocity, $v_{\mathrm{eq}} \sin i$, was determined by minimizing the artificial dark or bright bands in the Doppler maps that appear if the equatorial velocity, $v_{\text {eq }}$, is either too large or too small, respectively (for more details see Rice \& Strassmeier et al. 2000). Figure 8a demonstrates the basic effect that a wrong $v \sin i$ will impose onto the reconstruction. The best value from Sect. 4 was near $33 \mathrm{~km} \mathrm{~s}^{-1}$ with an error of just $0.5 \mathrm{~km} \mathrm{~s}^{-1}$. This is easily seen in the run of the minimum and maximum temperature and, of course, also in the $\chi^{2}$ variation, although not as dominant as for the temperature peaks. A value of $33.2 \pm 0.5 \mathrm{~km} \mathrm{~s}^{-1}$ (cf. Fig. 8a) seems the best value from the tests and agrees very well with previously published values from the literature.

\subsection{Inclination of the rotational axis}

In previous papers of this series the inclination of the rotational axis, $i$, was determined by reducing the misfit of the line profiles as a function of the inclination (e.g., Rice \& Strassmeier 2000). This method was also applied in the case of HD 31993, but due to repeated terminations of the iteration process of our test runs in the region of interest $\left(i>60^{\circ}\right)$ we were initially not sure whether the program had found the optimal solution. However, as it turned out, the program had repeatedly terminated the iterations because it had found the optimal solution much earlier in the iteration process than at other inclinations. By trial-and-error we found out that an $i$-value of $65^{\circ}$ yields the most "homogeneous" temperature maps. This finding makes us believe that $i=65^{\circ}$ is the most likely inclination angle with an uncertainty of approximately $\pm 15^{\circ}$.

\subsection{Photospheric temperature}

Despite the fact that the photospheric temperature is neither an input parameter nor kept constant during the line-profile inversion, the resulting maps indirectly depend on its initially chosen value. This is because of its non-linear relation on various other spectrum parameters that are kept unaltered during one inversion, most notably on the chemical abundances. Usually, we use the effective temperature as the temperature of the blank, i.e. spot free, star that is the starting point for the initial iteration. The test runs in Fig. 8c show the changes of the absolute and relative surface-temperature range along with the quality of the overall fit. Note that this figure is always only valid for a fixed abundance (in this case for the best-fit $\mathrm{Fe}$ and $\mathrm{Ca}$ abundances from Fig. 8d). Due to the comparably small differences of the obtainable $\chi^{2}$, we can state that our maps are not critically biased by the initially chosen input for the photospheric temperature. 


\section{Summary of parameter study}
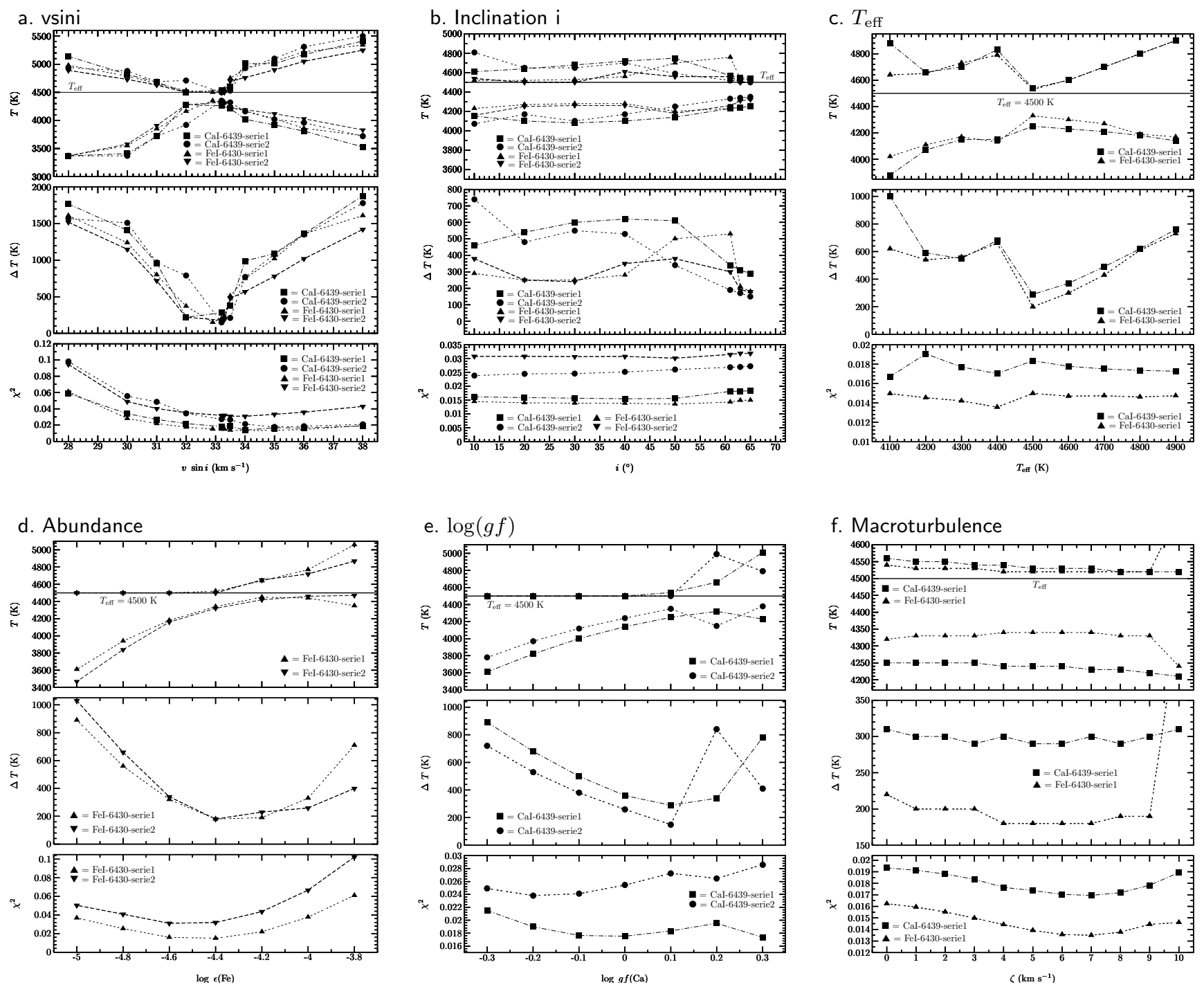

Fig. 8. A summary of the results from the parameter study. A single parameter is varied at a time while the others are kept constant at the values listed in Table 2. The parameters varied are: a) projected rotational velocity $(v \sin i)$, b) inclination of the rotation axis with respect to the line of sight $(i), \mathbf{c})$ the effective temperature of the $\left.\operatorname{star}\left(T_{\text {eff }}\right), \mathbf{d}\right)$ the chemical abundance (shown only for iron; $\log \epsilon$ ), e) the transition probability of the Ca I 643.9-nm line $(\log (g f))$, and f) the adopted radial-tangential macroturbulence $(\zeta)$. Each figure plots, from top to bottom, the upper and lower absolute temperature, $T$, (usually for both rotation series), the maximum temperature range in the map, $\Delta T$, (usually for Ca and Fe) and the quality of the fit expressed as $\chi^{2}$.

\subsection{Elemental abundances and log gf}

Figure $8 \mathrm{~d}$ shows the change of absolute minimum and maximum temperature, its difference, and the $\chi^{2}$ as a function of logarithmic abundance (only iron is shown). There is a broad but clear minimum from which the best-fit abundance can be determined. However, the effect of the chemical abundances on the line-profile reconstruction is not distinguishable from a change of the transition probability, $\log g f$, of the adopted spectral line (Fig. 8e), as was noted in previous papers in this series and quantified in tests with artificial data by Rice \& Strassmeier (2000). Also, abundances from just a single spectral line, even if completely deblended in the modelling as in the present study, can be quite uncertain and we emphasize that the abundances quoted in this paper are to be taken with care. Further uncertainty is introduced because the abundances also depend on the adopted effective temperature and microturbulence. However, our particular mapping technique bears - at least in principle - the capability to detect different abundances inside and outside of spotted regions.

\subsection{Micro- und macroturbulence}

Our first test was the recovery of the minimum- $\chi^{2}$ map with microturbulences, $\xi$, of $\pm 0.6 \mathrm{~km} \mathrm{~s}^{-1}$ around the literature value of $2.0 \mathrm{~km} \mathrm{~s}^{-1}$, in steps of $0.1 \mathrm{~km} \mathrm{~s}^{-1}$. The simulation confirmed 
the $2.0-\mathrm{km} \mathrm{s}^{-1}$ value to be the one with the best $\chi^{2}$ map, showing also the smallest range in temperature. Increased microturbulence causes increased minimum and maximum temperatures for $\mathrm{Ca}$ and $\mathrm{Fe}$ alike. Besides the simple $\chi^{2}$ statistics, the surface maps recovered showed easy-to-spot artifacts. At $\xi=$ $1.6 \mathrm{~km} \mathrm{~s}^{-1}$, i.e. $0.4 \mathrm{~km} \mathrm{~s}^{-1}$ below the best value, the "southern" hemisphere becomes warmer than the "northern" hemisphere by $\approx 500 \mathrm{~K}$ while the opposite is the case for $\xi=2.4 \mathrm{~km} \mathrm{~s}^{-1}$, i.e. $0.4 \mathrm{~km} \mathrm{~s}^{-1}$ above the best value.

In the second test series, the microturbulence was fixed at $2.0 \mathrm{~km} \mathrm{~s}^{-1}$ and the macroturbulence varied from $0 \mathrm{~km} \mathrm{~s}^{-1}$ (no macroturbulence) to $10 \mathrm{~km} \mathrm{~s}^{-1}$, in steps of $1 \mathrm{~km} \mathrm{~s}^{-1}$ (always assuming equal radial and tangential velocity components). The nominal value from the literature is $3.0 \mathrm{~km} \mathrm{~s}^{-1}$. Figure $8 \mathrm{f}$ shows the test results. While the maximum and minimum temperatures remained approximately constant for $\mathrm{Fe}$ and $\mathrm{Ca}$, respectively, the absolute minimum temperature was consistently cooler by $100 \mathrm{~K}$ for $\mathrm{Ca}$ but for the absolute maximum temperature agreed with the Fe values. This is easy to understand because the two lines are at different parts of their respective curves of growth. It is interesting to note though that the inversion from both species, Ca I and Fe I, showed a pronounced minimum in $\chi^{2}$ at approximately $7 \mathrm{~km} \mathrm{~s}^{-1}$, a value that is significantly larger than the literature value for "normal" stars (Gray 1992). Fekel (1997) had already suggested that active stars may have a larger macroturbulence. However, our finding remains inconclusive because we could partly "compensate" the effect of a higher macroturbulence with an alteration of the microturbulence.

\section{Discussion and summary}

The Hipparcos parallax puts HD 31993 at a position in the HR-diagram where a deep convective envelope coexists with a hydrogen-burning shell and rapid redistribution of moment of inertia must occur or had occurred (e.g. Schrijver \& Pols 1993; doNascimento et al. 2000). This is thought to be a time of high dynamo efficiency due to extra shear forces within the bottom layers of the convective envelope and induces rotational mixing (Palacios et al. 2003). It also marks the end of the first Li dredge-up according to the models of Charbonnel \& Balachandran (2000). While a comparison with the Schaller et al. (1992) "overshooting" models suggests a mass of $1.9 \pm$ $0.3 M_{\odot}$, Charbonnel \& Balachandran (2000) obtain 2.2 $\pm 0.6 M_{\odot}$ from a comparison with their own tracks. Therefore, it is currently not possible to conclusively decide whether HD 31993 has a degenerate helium core and, thus, later undergoes a Heflash or will evolve on to the early AGB and experience a second Li dredge-up. However, it seems clear that HD 31993 is in an evolutionary episode where it warrants a search for a relation between its magnetic surface activity and the $\mathrm{Li}$ abundance. A Doppler-imaging study of the surface Li abundance and its possible inhomogeneity would be most useful.

Previous maps of rapidly-rotating late-type giants show a large spread of spot morphology (a summary of the literature was given by Strassmeier 2002 and references therein). Out of the total of 13 mapped post-main-sequence stars of spectral type G8-K2 and luminosity class III or III-IV, seven had a "polar spot": UZ Lib (K0III, $P_{\text {rot }}=4.8$ days), DM UMa (K0-1III-IV, $P=7.5$ days), YY Men (K2III, $P=$ 9.5 days), HU Vir (K0III-IV, $P=10.4$ days), IL Hya (K0IIIIV, $P=12.7$ days), CM Cam (G8II-III, $P=16.0$ days), and KU Peg (G9II-III, $P=25.0$ days). High-latitude spots were found on 4 others: on IM Peg (K2III, $P=24.6$ days), HK Lac (K0III, $P=24.4$ days), XX Tri (K0III, $P=24.0$ days), and HD 208472 (G8III, $P=22.4$ days). Two K giants without polar or very high-latitude spots were found as well: $\sigma$ Gem (K1III, $P=19.6$ days) and our new target HD 31993 (K2III, $P=25.3$ days).

Only three of the above 13 giants are not in a close binary where the high rotational angular velocity is maintained by the orbital momentum. Obviously, the two rapidly-rotating single giants YY Men and HD 31993, as well as the one effectively-single giant CM Cam (a component in a wide binary), must have had another angular-momentum history. Therefore, Doppler maps from binary giants and from single giants are likely not directly comparable. Our detection of differential rotation is also the first detection for a single rapidlyrotating active giant, but again not directly comparable to results from binaries.

The diverging observations - stars with or without a polar spot, with or without detected differential rotation and of solar or anti-solar direction - pose no basic problem for a model that describes the deflection of rising magnetic flux tubes due to Coriolis forces (e.g. Schüssler \& Solanki 1992; Granzer et al. 2000) and thus explains the existence of surface spots on single stars and binaries as well. However, the model appears to miss a physical ingredient that could also cause, but not for all evolutionary stages, a pole-ward deflection in the absence of significant Coriolis forces. We speculate that an equator-ward meridional flow at the bottom of the convective envelope may act as the extra mixing needed for the Li dredge-up while, at the same time, its opposite flow direction on the stellar surface will sweep magnetic flux to high latitudes and eventually to the rotational poles. Differential rotation may play the second key role and its anti-solar direction may be interpreted as indication for anti-solar meridional circulation, i.e. directed towards the poles at the surface and vice versa at the bottom of the convection zone. We conclude that monitoring the spatially resolved structures of active stars for many epochs has the potential to resolve some of the basic questions of solar and stellar magnetic activity.

Acknowledgements. KGS is very grateful to the German Science Foundation (DFG) for support under grant STR645/1. Special thanks are due to John Rice for maintaining the TempMap code and numerous discussions concerning its application to cool stars. We also thank an anonymous referee for his/her helpful comments.

\section{References}

Balachandran, S. C., Fekel, F. C., Henry, G. W., \& Uitenbroek, H. 2000, ApJ, 542, 978

Balona, L. A. 1987, SAAO Circ., 11, 1

Beavers, W. I., \& Eitter, J. J. 1986, ApJS, 62, 147

Bidelman, W. P., \& MacConnell, D. J. 1973, AJ, 78, 687 
Castilho, B. V., Gregorio-Hetem, J., Spite, F., Barbuy, B., \& Spite, M. 2000, A\&A, 364, 674

Castilho, B. V., Spite, F., Barbuy, B., et al. 1999, A\&A, 345, 249

Charbonnel, C., \& Balachandran, S. C. 2000, A\&A, 359, 563

Collier Cameron, A. 2002, AN, 323, 336

Collier Cameron, A., \& Donati, J.-F. 2002, MNRAS, 329, L23

De Medeiros, J. R., \& Mayor, M. 1999, A\&AS, 139, 433

do Nascimento, J. D., Charbonnel, C., Lébre, A., de Laverny, P., \& De Medeiros, J. R. 2000, A\&A, 357, 931

ESA 1997, The Hipparcos and Tycho catalog, ESA SP-1200

Fekel, F. C. 1997, PASP, 109, 514

Fekel, F. C., \& Balachandran, S. C. 1993, ApJ, 403, 708

Fekel, F. C., Moffett, T. J., \& Henry, G. W. 1986, ApJS, 60, 551

Flower, P. J. 1996, ApJ, 469, 355

Gezari, D. Y., Pitts, P. S., \& Schmitz, M. 1999, Catalog of Infrared Observations, Edition 5, CDS

Granzer, T., Schüssler, M., Caligari, P., \& Strassmeier, K. G. 2000, A\&A, 355, 1087

Gray, D. F. 1992, The observation and analysis of stellar photospheres, CUP (Cambridge)

Grevesse, N., \& Sauval, A. J. 1998, Space Sci. Rev., 85, 161

Grevesse, N., \& Sauval, A. J. 1999, A\&A, 347, 348

Hooten, J. T., \& Hall, D. S.1990, ApJS, 74, 225

Huenemoerder, D. P., \& Ramsey, L. W. 1987, ApJ, 319, 392

Israelian, G., Santos, N. C., Mayor, M., \& Rebolo, R. 2001, Nature, 411, 163

Kővári, Zs., Strassmeier, K. G., Bartus, J., et al. 2001, A\&A, 373, 199

Kratzwald, L. 2003, Masters Thesis, Univ. Vienna

Kurucz, R. L. 1993, ATLAS-9, CD-ROM No. 13

Lloyd-Evans, T., \& Koen, M. C. J. 1987, SAAO Circ., 11, 21

Micela, G., Favata, F., \& Sciortino, S. 1997, A\&A, 326, 221

Palacios, A., Talon, S., Charbonnel, C., \& Forestini, M. 2003, A\&A, 399,603
Piskunov, N. E., \& Rice, J. B. 1993, PASP, 105, 1415

Piskunov, N. E., Tuominen, I., \& Vilhu, O. 1990, A\&A, 230, 363

Ramaty, R., Tatischeff, V., Thibaud, J. P., Kozlovsky, B., \& Mandzhavidze, N. 2000, ApJ, 534, L207

Rice, J. B. 2002, AN, 323, 220

Rice, J. B., \& Strassmeier, K. G. 2000, A\&AS, 147, 151

Rice, J. B., Wehlau, W. H., \& Khokhlova, V. L. 1989, A\&A, 208, 179

Scarfe, C. D., Batten, A. H., \& Fletcher, J. M. 1990, Publ. Dominion Astron. Obs. Victoria, 18, 21

Schaller, G., Schaerer, D., Meynet, G., \& Maeder, A. 1992, A\&AS, 96, 269

Schrijver, C. J., \& Pols, O. R. 1993, A\&A, 278, 51

Schüssler, M., \& Solanki, S. K. 1992, A\&A, 281, L69

Slee, O. B., Nelson, G. J., Stewart, R. T., et al. 1987, MNRAS, 229, 659

Sperl, M. 1998, in Commun. Asteroseismology, ed. M. Breger, IfA Vienna, vol. 111

Spite, M., Maillard, J. P., \& Spite, F. 1984, A\&A, 141, 56

Strassmeier, K. G. 1999, A\&A, 347, 225

Strassmeier, K. G. 2002, AN, 323, 309

Strassmeier, K. G., Bartus, J., Cutispoto, G., \& Rodonó, M. 1997a, A\&AS, 125, 11

Strassmeier, K. G., Boyd, L. J., Epand, D. H., \& Granzer, T. 1997b, PASP, 109, 697

Strassmeier, K. G., Fekel, F. C., Bopp, B. W., Dempsey, R. C., \& Henry, G. W. 1990, ApJS, 72, 191

Strassmeier, K. G., \& Rice, J. B. 2003, A\&A, 399, 315

Strassmeier, K. G., Serkowitsch, E., \& Granzer, T. 1999, A\&AS, 140, 29

Weber, M., \& Strassmeier, K. G. 1998, A\&A, 330, 1029

Weber, M., \& Strassmeier, K. G. 2001, A\&A, 373, 974 